\title{
HISTOGRAM OF ORIENTED GRADIENTS WITH CELL AVERAGE BRIGHTNESS FOR HUMAN DETECTION
}

\section{Marek Wójcikowski}

Gdańsk University of Technology, Faculty of Electronics, Telecommunications and Informatics, G. Narutowicza 11/12, 80-233 Gdańsk, Poland $\triangle$ wujek@ue.eti.pg.gda.pl,+4858347 1974)

\begin{abstract}
A modification of the descriptor in a human detector using Histogram of Oriented Gradients (HOG) and Support Vector Machine (SVM) is presented. The proposed modification requires inserting the values of average cell brightness resulting in the increase of the descriptor length from 3780 to 3908 values, but it is easy to compute and instantly gives $\approx 25 \%$ improvement of the miss rate at $10^{-4}$ False Positives Per Window (FPPW). The modification has been tested on two versions of HOG-based descriptors: the classic Dalal-Triggs and the modified one, where, instead of spatial Gaussian masks for blocks, an additional central cell has been used. The proposed modification is suitable for hardware implementations of HOG-based detectors, enabling an increase of the detection accuracy or resignation from the use of some hardware-unfriendly operations, such as a spatial Gaussian mask. The results of testing its influence on the brightness changes of test images are also presented. The descriptor may be used in sensor networks equipped with hardware acceleration of image processing to detect humans in the images.
\end{abstract}

Keywords: digital image processing, object detection, human detection.

(C) 2016 Polish Academy of Sciences. All rights reserved

\section{Introduction}

Detection of persons in images is an important and challenging task needed for applications such as driving assistance, autonomous driving or video surveillance, where the pedestrian detection must be both robust and in real-time. There are two main methods of person detection: the single-scanning window and the part-based detector. Scanning window methods are based on various feature descriptors, such as Histogram of Oriented Gradients (HOG) [1], Haar wavelet [2], Edge Orientation Histogram (EOH) [3, 4], and Local Binary Pattern (LBP) [3, 4]. The descriptors are classified by using machine learning techniques, such as Support Vector Machine (SVM) [5,6] or a boosting classifier. The SVM is a well-known method of classification with a solid mathematical background, where the learning phase is reasonably short, but the classification stage requires a significant number of multiplications and additions. The SVM has been successfully applied to many different problems $[7,8]$. The boosting classifier consists of a cascade of "weak" classifiers, where early stages of the cascade reject most negative data. Owing to this, only a limited number of samples traverse the full cascade, thus this method requires a very long time in the learning stage and it is quick in the classification stage. Part-based methods [9] mainly use a deformable model, which improves the detection performance. They generally work better at partial occlusions and for pedestrians in various poses. Feature descriptors are used for detection of parts of the model. The part-based detectors require increased computational costs and it is more difficult to implement a real-time robust application, while many successful real-time applications using a sliding window have been reported. 
Among various feature descriptors, the HOG descriptor outperforms most other techniques and is widely used for pedestrian detection. The HOG descriptor used together with the SVM classifier is one of the best-known human detection methods. The HOG has been introduced in [10] and [1]; many modifications of it may be found in the literature, intended for improvement of the detection quality or speed. Combining the HOG descriptor with boosting-based methods gives higher classification speeds than the SVM-based methods [11, 12]. In [13], the HOG descriptor combined with Haar-like features and the boosted cascade classifier has been proposed to obtain a better detection accuracy and efficiency. In [14], the HOG descriptor is combined with multi-scale curvelet features for the full body detection. The simultaneous use of HOG and LBP gives long descriptors but it also gives very good detection results, as described in [15] and [16]. Zeng et al. [17] use a two-stage cascade of rejecters: the HOG classifiers and LBP classifiers, to improve processing long descriptors.

To decrease the processing time and achieve real-time operation, many algorithm modifications and acceleration techniques have been invented. A widely-used technique is the integral image method [18], which provides the possibility of obtaining area-based descriptor values in a constant time. Hardware implementations, such as the feature extraction accelerator VLSI [19], can be used in portable, on-board vehicle systems or sensor networks; hardware accelerators can also increase the efficiency of computer-based solutions. However, not all algorithms can be efficiently implemented in hardware, therefore the researchers are searching for pipeline-friendly methods, which can be embedded into the vision chips [20].

In this paper, a modification of the classic HOG descriptor is proposed. The main contribution of this paper is the introduction of additional, easily calculated values to the descriptor, which instantly improves the miss rate parameter of window detector. To provide clarity of the presented evaluations, all results are compared with those of the well-known and well-described classic method presented in [1] and are tested using the INRIA dataset [21].

\section{The descriptor}

Calculation of the classic HOG descriptor begins with dividing an image under the detection window into a dense grid of rectangular cells. For each cell a separate orientation of gradients is calculated. The histogram consists of evenly spaced orientation bins accumulating the weighted votes of gradient magnitude of each pixel belonging to the cell. In [1], $8 \times 8$ pixel cells and 9 bins for the orientation range of 0-180 degrees have been used. Additionally, the cells are grouped into blocks and for each block all cell histograms are normalised. The blocks are overlapping, so the same cell can be differently normalised in several blocks. The descriptor is calculated using all overlapping blocks from the image detection window. From the detection window of size $64 \times 128$ pixels and for a block of $2 \times 2$ cells, shifted by 8 pixels, 3780 features per detection window are obtained.

The basic version of HOG descriptor would not give such good results, unless some additional techniques are used, as proposed by Dalal and Triggs [1]:

- For colour images, separate gradients for each colour are calculated, but only the gradient with the largest norm is used.

- A Gaussian mask is used on each pixel of the block to down-weight the pixels near the edges.

- Each vote of gradient magnitude is bi-linearly interpolated into neighbouring bins and in the same way is also divided into neighbouring cells. This procedure is called a tri-linear interpolation.

In this paper, a modification of the classic HOG descriptor is proposed, where an additional value $I_{i}$ is included in each cell $i$ in the descriptor, as shown in Fig. $1 . I_{i}$ is the average brightness of the cell $i$, calculated using an average of $R, G$ and $B$ pixel components. Using the infinity norm of $R, G$ and $B$, instead of an average for calculating a cell's average brightness, gives 
slightly worse results. Calculation of $I_{i}$ can be done easily during calculation of the histograms of gradients; the main disadvantage is the increase of the descriptor length from 3780 to 3908 features (for the detection window of size $64 \times 128$ pixels and for a block of $2 \times 2$ cells, shifted by 8 pixels), which can cause an increased processing time. It is also possible to use $I_{i}^{\prime}=I_{i}-I_{\text {avg }}$ instead of $I_{i}$, where $I_{a v g}$ is the average brightness of the window, but the test results are similar to those using $I_{i}$.
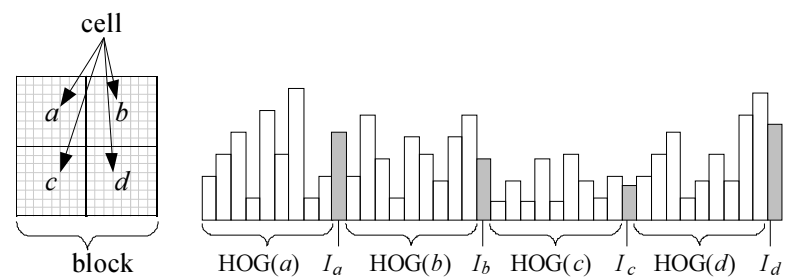

Fig. 1. The proposed structure of descriptor with the average brightness value of each cell. $I_{i}$ represents the average intensity of pixels belonging to the cell $i=\{a, b, c, d\}$.
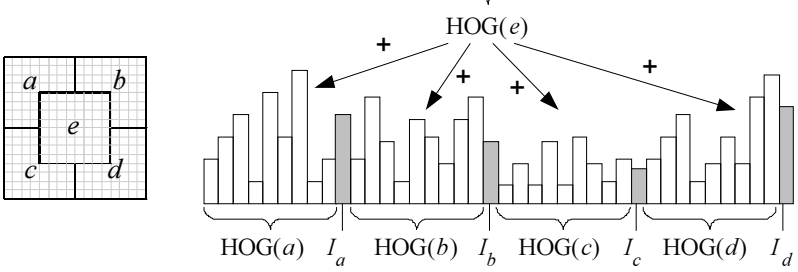

Fig. 2. The structure of descriptor using the central cell approach applied in [16], instead of a Gaussian spatial mask used in [1], with the cells' average brightness values included in the descriptor.

In hardware implementation, the most challenging operations needed for calculating the HOG are: the Gaussian mask and the tri-linear interpolation, since they do not fit well in a pipelined style of hardware operation and integral image approach. In [16] an additional HOG of the cell centred in the original block has been used to replace the spatial pixel weighting, which in fact improved the overall detection quality. The proposed modification of adding a brightness-based value has also been tested with the central cell approach presented in [16] instead of the Gaussian spatial mask, which enables easier hardware implementation.

\section{Learning and testing the classifiers}

The proposed modification of HOG descriptor has been used with the linear SVM for classification of the analysed images. The linear SVM is based on solving the optimisation problem $[5,6]$ :

$$
\min _{\mathbf{w}, b, \boldsymbol{\xi}} \frac{1}{2} \mathbf{w}^{T} \mathbf{w}+C \sum_{i=1}^{L} \xi_{i},
$$

subject to:

$$
y_{i}\left(\mathbf{x}_{i} \cdot \mathbf{w}+b\right)-1+\xi_{i} \geq 0 \quad \xi_{i} \geq 0, \quad i=1, \ldots, L,
$$


where $\left\{\mathbf{x}_{i}, y_{i}\right\}$ is the training data set of size $L$ and $\mathbf{x} \in \mathfrak{R}^{D}$ is the input point of $D$ attributes with the corresponding label $y_{i}=-1$ or $+1 . \xi_{i}$ is a positive slack variable, which relaxes the constraints and allows for misclassifying some points, when the problem is not fully linearly separable. The variables $\mathbf{w}$ and $b$ define the optimal orientation of the hyperplane, separating the points belonging to two different classes with the soft error margin controlled by the parameter $C$. The minimisation problem (1), (2) is solved using the iterative quadratic problem solver, where the termination criteria for the algorithm may be: the maximum number of iterations and/or the tolerance error $\varepsilon$. The HOG descriptor has been combined with the linear SVM to obtain a classifier. In this paper the value of the parameter controlling the error margin in SVM has been set to $C=0.01$, which enables easy comparison with the results presented in [1], where the same value for $C$ has been applied. For the experiments, the SVM implementation from OpenCV library version 2.4.11.0 has been used, with the termination criterion $\varepsilon=0.001$.

The SVM has been trained using the INRIA data set (2416 positive examples and 24360 negative examples, including their mirrored versions) in the same way as in [1], i.e. the retraining phase has been completed using hard training examples detected after the first training. The examples of the training images are shown in Fig. 3.
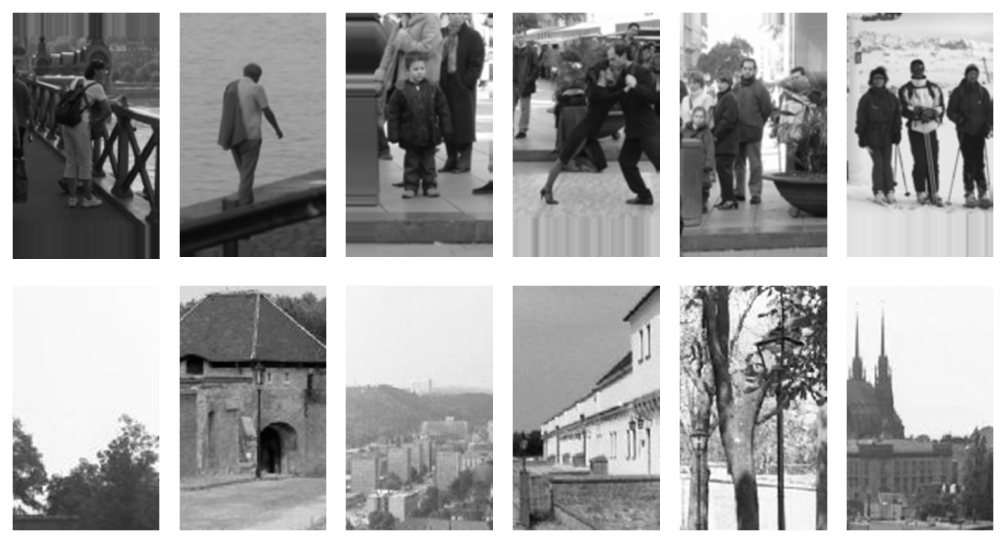

Fig. 3. A few examples of positive images containing pedestrians (top row) and negative, nonpedestrian images (bottom row) from the INRIA database [21].

For the testing, the INRIA test images have been used with 1132 positive examples and 453 non-pedestrian negative images, where each negative image has been extensively searched with 8 pixel shift of the test window and $1.2 \mathrm{x}$ scale down factor of the image. The testing procedure was the same as in [1]. Usually the Receiver Operating Characteristics (ROC) curves are used to quantify the performance of detectors, based on the classification return values, which are the signed distances to the margin in the 2-class SVM classifier. The shape of ROC curves does not enable easy distinguishing of small probabilities, so in the further considerations the RecallPrecision (RP) and Detection Error Tradeoff (DET) curves will be used, which contain the same information as the ROC curves. The RP curve plots precision versus recall on a log-log scale. Precision and recall are defined as:

$$
\begin{gathered}
\text { Precision }=\frac{T P}{T P+F P}, \\
\text { Recall }=\frac{T P}{T P+F N},
\end{gathered}
$$

where: $T P$ - the number of windows where there was a person and a person has been detected; 
$F P$ - the number of windows where there was no person, but the detector indicated the presence of a person; $T N$ - the number of windows where there was no person and the detector did not detect any person; $F N$ - the number of windows where there was a person but the detector did not detect any person.

The RP curves are presented in Fig. 4 for three classifiers: the Dalal-Triggs classifier from [1] (HOG) and the classifiers from Fig. 1 and Fig. 2.

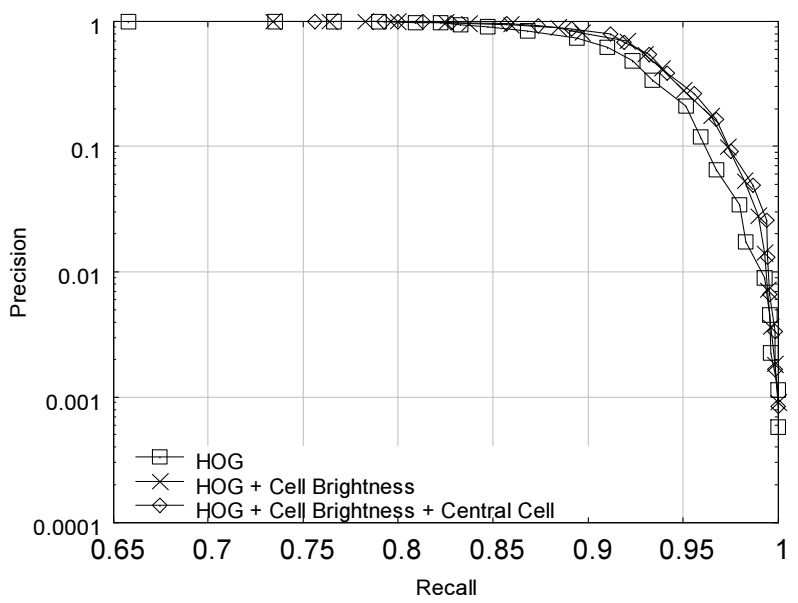

Fig. 4. Recall-Precision curves for the classifier using the descriptor from Fig. 1 (HOG + Cell Brightness) and from Fig. 2 (HOG + Cell Brightness + Central Cell). For comparison, the results from the Dalal-Triggs method [1] are also given (HOG). All tests have been performed using the INRIA Person dataset with re-training on hard examples. The negative test images have been scanned using $1.2 \mathrm{x}$ rescale factor and 8-pixel window shift (the same procedure as described in [1]).

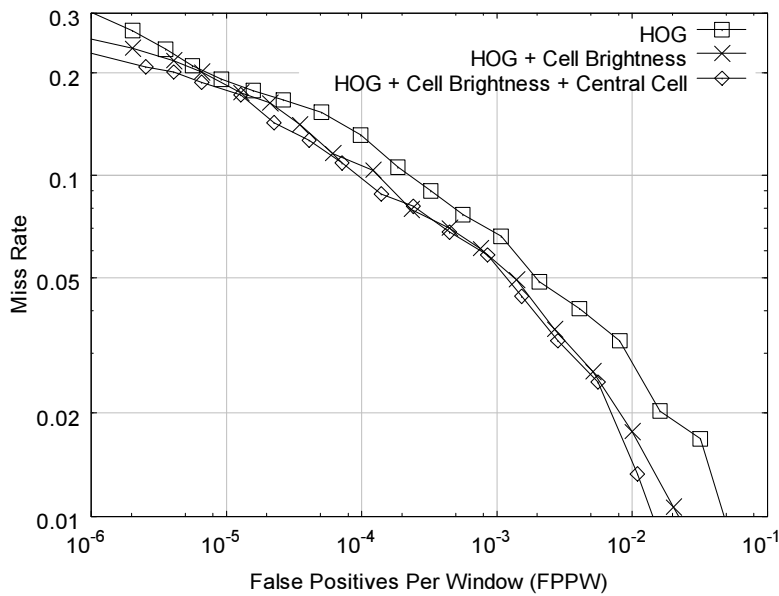

Fig. 5. Detection Error Trade-off curves for the classifier using the descriptor from Fig. 1 (HOG + Cell Brightness) and from Fig. 2 (HOG + Cell Brightness + Central Cell). For comparison, the results from the Dalal-Triggs method [1] are also given (HOG). All tests have been performed using the INRIA Person dataset with re-training on hard examples. The negative test images have been scanned using 1.2x rescale factor and 8-pixel window shift (the same procedure as described in [1]). 
In Fig. 5 the DET curves are shown, which use the measures: the MissRate and False Positives Per Window (FPPW), defined as follows:

$$
\begin{gathered}
\text { MissRate }=1-\text { Recall }=\frac{F N}{T P+F N}, \\
F P P W=\frac{F P}{F P+T N} .
\end{gathered}
$$

The test results presented in Fig. 5 show $\approx 25 \%$ improvement of the miss rate at $10^{-4}$ FPPW for the proposed descriptor, which is equivalent to three times better FPPW at the same miss rate. It must be noted that part of the miss rate improvement has been achieved by using the central cell approach.

To seek an optimal value of the parameter $C, k$-fold cross-validation with the INRIA train dataset and $k=9$ has been applied, where $C$ has been raised from the value $C_{0}=2 \cdot 10^{-4}$ up to $C_{j}=20$ with step $S=1.2$ in iterations $j=0,1, \ldots, n$, where each next value of $C_{j}$ has been calculated as:

$$
C_{j+1}=S \cdot C_{j}
$$

As the result of this search, the new value of $C=0.03125$ has been obtained, for which the test set error is minimal. However, the performance of the classifiers with the new value of $C$ was similar to the previous results (see Fig. 6), therefore the previous value $C=0.01$ has been used in all further calculations presented in this paper.

a)

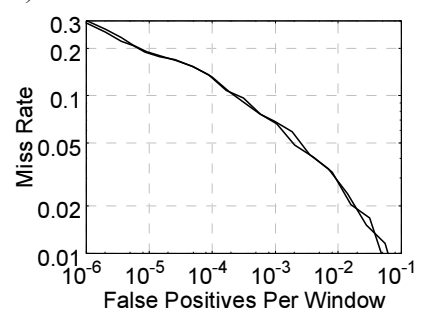

b)

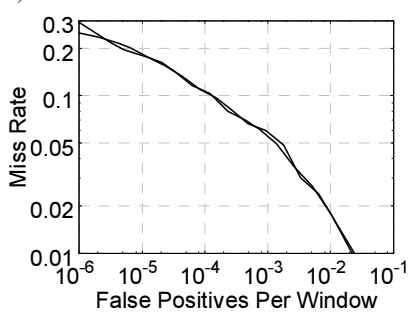

c)

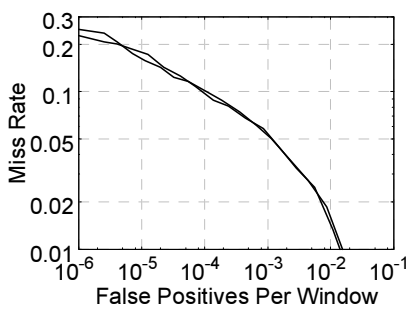

Fig. 6. Detection Error Trade-off curves for the classifiers learned with the SVM's parameter value $C=0.01$ (dashed line) and the value $C=0.03125$ obtained from $k$-fold cross-validation and sweeping (solid line) using: a) the descriptor from the Dalal-Triggs method [1]; b) the descriptor from Fig. 1; c) the descriptor from Fig. 2.

Using brightness values directly in the descriptor might suggest that the descriptor has lost its brightness-invariance. To test the behaviour of the descriptor, a test set has been prepared, containing the transformed INRIA test images, where the intensities of the positive test images have been randomly changed according to the equation:

$$
\mathbf{I}_{\text {new }}=\alpha \mathbf{I}+\beta,
$$

where: I and $\mathbf{I}_{n e w}$ - the intensities of the image before and after transformation, respectively; $\alpha$, $\beta$ - the coefficients, randomly changed for each image, $\alpha$ has been changed in the range from 0.5 to 3 and $\beta$ from -50 to 100 .

The results of the test using the brightness-transformed positive test images are presented in Fig. 7. 

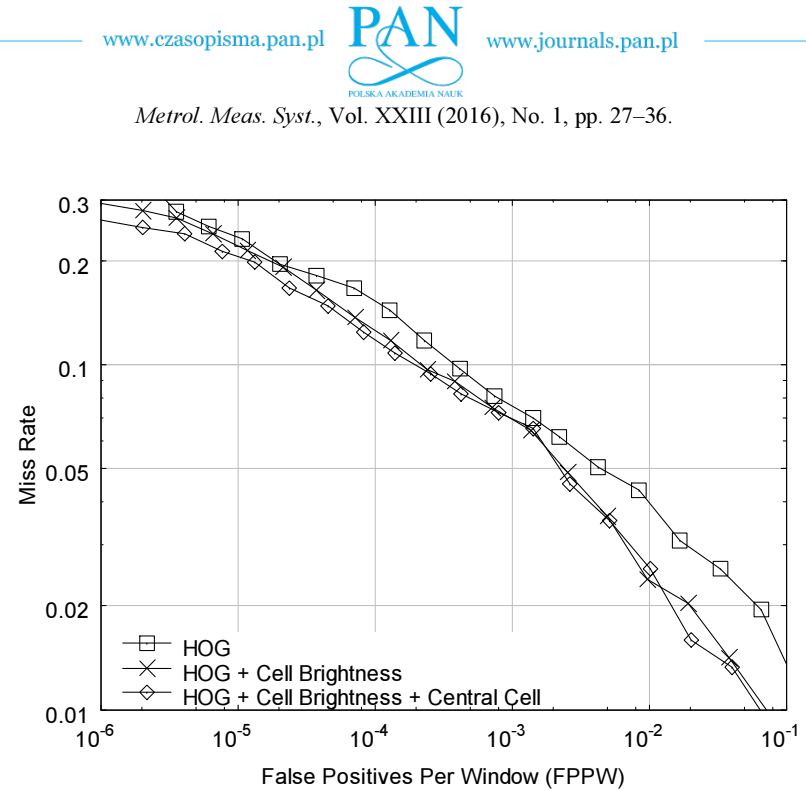

Fig. 7. The test results showing the influence of brightness changes according to (8) for the proposed descriptor from Fig. 1 (HOG + Cell Brightness) and from Fig. 2 (HOG + Cell Brightness + Central Cell).

For comparison, the results from the Dalal-Triggs descriptor [1] are also given (HOG). Training and testing have been performed on the INRIA Person dataset using the same procedure as described in [1] with randomly changed brightness of the positive test images.

In another test, instead of uniformly changing the brightness of the whole window, the brightness has been randomly changed at random regions of the image. First, each tested window has been divided into two parts by a diagonal line at a variable position and angle, then the brightness of a randomly chosen part has been changed according to (8) with the coefficients changed randomly for each window. The random brightness change has been also applied to the randomly selected rectangular areas of the window (with the random number of rectangles from 1 to 4). The examples of images after those transformations are shown in Fig. 8. The DET curves presenting the performance of detectors for the manipulated images are shown in Fig. 9.

The results from both tests with the use of images with randomly changed brightness show that the DET curves in Fig. 7 and Fig. 9 are always above the DET curve for the classic HOG, proving that the proposed modification gives better results than the classic HOG descriptor.
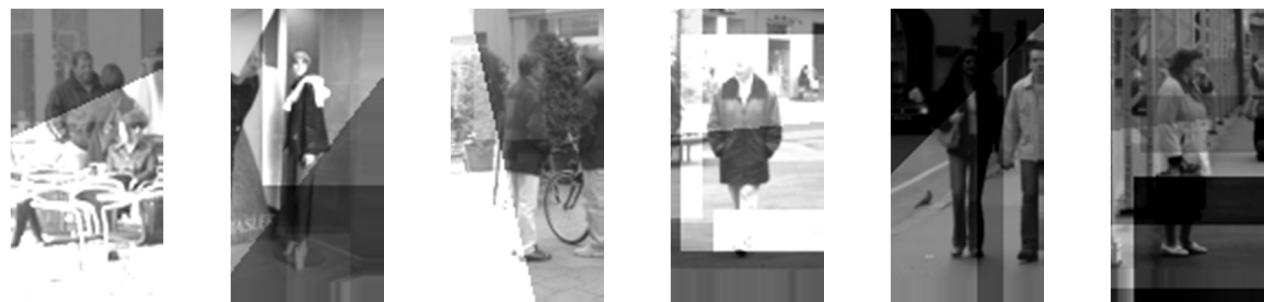

Fig. 8 Examples of the positive test images from the INRIA database with randomly changed brightness in random areas. 


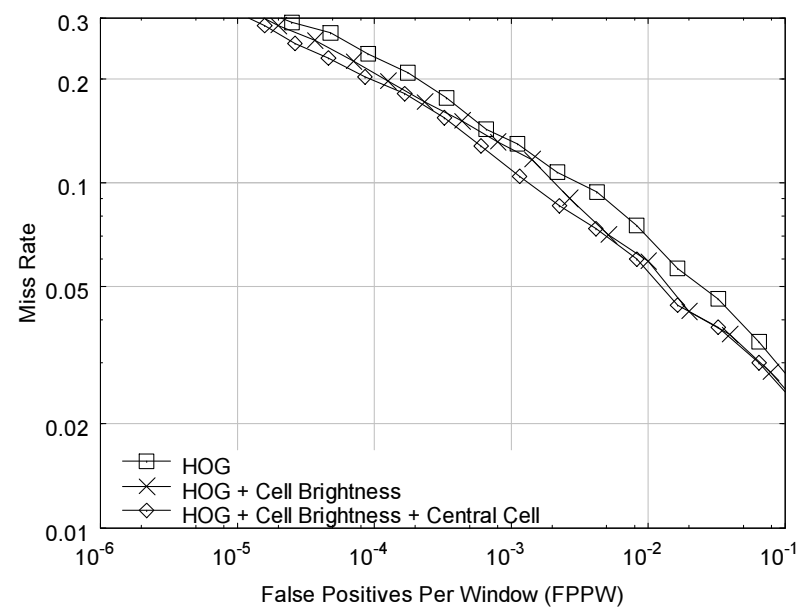

Fig. 9. The test results showing the influence of random brightness change in random rectangular regions for the proposed descriptor from Fig. 1 (HOG + Cell Brightness) and from Fig. 2 (HOG + Cell Brightness + Central Cell). For comparison, the results from the Dalal-Triggs descriptor [1] are also given (HOG). Training and testing have been performed on the INRIA Person dataset using the same procedure as described in [1], where the positive test images have randomly changed brightness of randomly selected regions of the image.

The calculation times of the proposed solution are presented in Table 1. Due to the inclusion of the brightness data into the descriptor from Fig. 1, the classification time of a single detection window (measured as the pure classification time, not including the time spent on reading the image from disk and writing the results) has increased by approx. $1 \%$ comparing with that for the classic HOG descriptor. The descriptor from Fig. 2 is by $\approx 2 \%$ slower, but it has the potential to be more effective in hardware, pipelined implementation.

Table 1. Comparison of the calculation times of SVM implementations. The descriptors have been written in $\mathrm{C}++$ using Open $C V$ version 2.4.11.0 of the SVM libraries. The evaluations have been made using a PC computer with Intel i7 $3.2 \mathrm{GHz}$ and 64-bit Windows 7 operating system. Pixel intensity values have been represented as 8-bit unsigned numbers. For the magnitude of the gradient, 64-bit floats have been used; the angle has been calculated in degrees and saved as a 32-bit integer. The histograms have been represented as STL vectors of 64-bit floating-point numbers.

\begin{tabular}{|l|r|r|r|r|}
\hline & Units & $\begin{array}{c}\text { Dalal-Triggs } \\
\text { HOG } \\
\text { descriptor } \\
\text { [1] }\end{array}$ & $\begin{array}{r}\text { HOG + Cell } \\
\text { Brightness } \\
\text { descriptor } \\
\text { from Fig. 1. }\end{array}$ & $\begin{array}{c}\text { HOG + Cell } \\
\text { Brightness + } \\
\text { Central Cell } \\
\text { descriptor from } \\
\text { Fig. 2. }\end{array}$ \\
\hline Descriptor length (the number of values) & - & 3780 & 3908 & 3908 \\
\hline Average classification time of single test window & {$[\mathrm{ms}]$} & 4.562 & 4.605 & 4.650 \\
\hline $\begin{array}{l}\text { Total learning time (26776 images), including reading } \\
\text { the image files from disk, calculation of the descriptor } \\
\text { and saving the results to disk }\end{array}$ & {$[\mathrm{s}]$} & 820 & 778 & 708 \\
\hline $\begin{array}{l}\text { Total classification time (10192 images), including } \\
\text { reading the image files from disk, calculating the } \\
\text { descriptor and saving the results to disk }\end{array}$ & {$[\mathrm{s}]$} & 95.7 & 96.0 & \\
\hline
\end{tabular}

Operation of the descriptor is shown in Fig. 10, where a dense scan of an image containing standing persons has been made. As can be seen, the detector using the proposed descriptor gives more $T P$ detections for almost each person on the image. At the same time, a few FP 
detections are skipped - in Fig. 10b the first person to the left and the first persons to the right have some false detections, which are correctly not detected in Fig. 10d.

a)

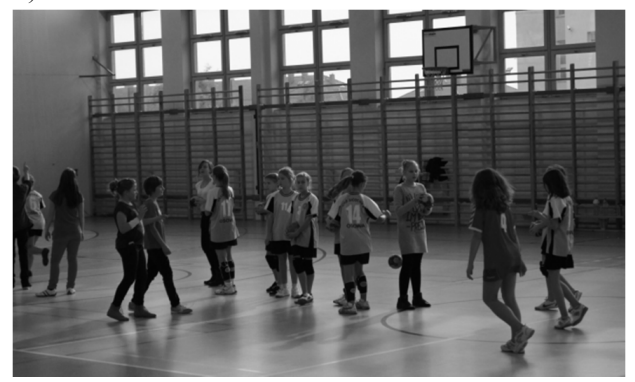

c)

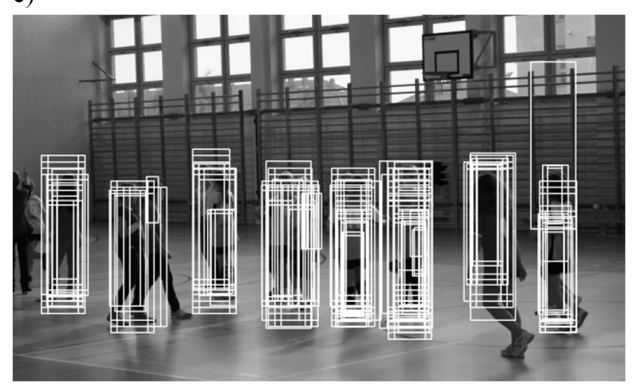

b)

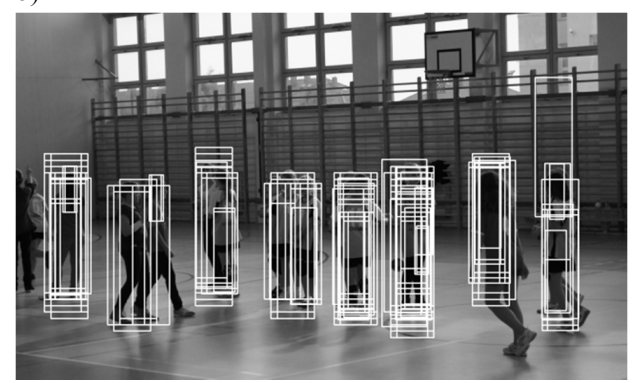

d)

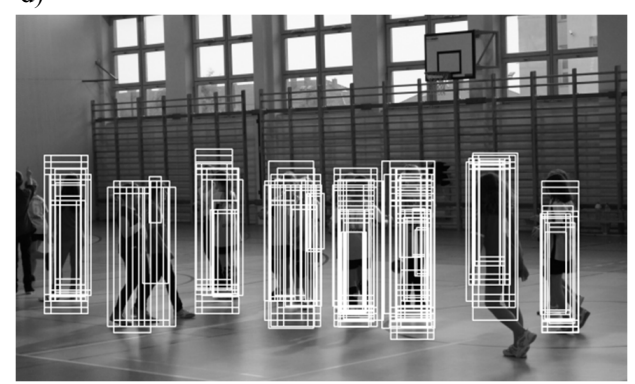

Fig. 10. An example of dense scan detection results: a) the input image; b) the detection results from the Dalal-Triggs's descriptor; c) the detection results from the descriptor from Fig. 1; d) the detection results from the descriptor from Fig. 2. The overlapping detections have not been merged (i.e., using non-maximum suppression) to show all detections. The image of size $1474 \times 828$ pixels has been scanned with the moving window with the step of 3 pixels and rescaled down with the factor 1.1 .

\section{Conclusions}

The main contribution of the author is the idea of using additional information in the HOG descriptor based on average pixel intensity. This simple modification slightly increases the length of the descriptor but it results in a significant improvement of the miss rate of the window detector. The proposed idea has been compared with the well-known HOG descriptor described in [1], also the modification based on the idea of using a central cell instead of a spatial Gauss mask [16] has been made. The test results show that this modification gives valuable hints to the SVM classifier, resulting in the miss rate improvement by $\approx 25 \%$ at $10^{-4} \mathrm{FPPW}$ over the original version of the HOG method, at the expense of up to $\approx 2 \%$ increase of the calculation time. Due to a shallow shape of DET curves, such an improvement in the miss rate is equivalent to 3 times better FPPW at the same miss rate. It has also been shown that the proposed modification, despite the fact that it uses the pixel intensities in the descriptor in addition to gradients, it still provides the improvement for images with randomly changed brightness. This shows that the brightness values present in the proposed descriptor contain additional information that helps the SVM to discriminate between positive and negative samples and may be considered as another procedure to improve the miss rate of the detectors. It is highly probable that adding the brightness-based values can improve many other descriptors based on the HOG method. 


\section{References}

[1] Dalal, N., Triggs, B. (2005). Histograms of oriented gradients for human detection. Proc. IEEE Int. Conf. Comput. Vision Pattern Recognit., 886-893.

[2] Viola, P., Jones, M. (2001). Rapid object detection using a boosted cascade of simple features. Proc. IEEE Int. Conf. Comput. Vision Pattern Recognit., 511-518.

[3] Ma, Y., Chen, X., Jin, L., Chen, G. (2011). A monocular human detection system based on EOH and oriented LBP features. Proc. 7th Int. Conf. Adv. Visual Comput., I, 551-562.

[4] Ma, Y., Deng, L., Chen, X., Guo, N. (2013). Integrating Orientation Cue With EOH-OLBP-Based Multilevel Features for Human Detection. IEEE Trans. Circuits Syst. Video Technol., 23(10), 1755-1766.

[5] Boser, B.E., Guyon, I., Vapnik, V. (1992). A training algorithm for optimal margin classifiers. Proc. Fifth Annual Workshop on Computational Learning Theory, ACM Press, 144-152.

[6] Cortes, C., Vapnik, V. (1995). Support-vector network. Machine Learning, 20, 273-297.

[7] Cui, J., Wang, Y., (2010). A novel approach of analog fault classification using a Support Vector Machines classifier. Metrol. Meas. Syst., 17(4), 561-581.

[8] Wójtowicz, B., Dobrowolski, A., Tomczykiewicz, K., (2015). Fall detector using discrete wavelet decomposition and SVM classifier. Metrol. Meas. Syst., 22(2), 303-314.

[9] Zhang, H., Bai, X., Zhou, J., Cheng, J., Zhao H. (2013). Object Detection via Structural Feature Selection and Shape Model. IEEE Trans. Image Process., 22(12), 4984-4995.

[10] Lowe, D.G. (2004). Distinctive Image Features from Scale-Invariant Keypoints. Int. Journal of Comput. Vision, 60(2), 91-110.

[11] Zhu, Q., Yeh, M.C., Cheng, K.T., Avidan, S. (2006). Fast human detection using a cascade of histograms of oriented gradients. Proc. IEEE Int. Conf. Comput. Vision Pattern Recognit., 2, 491-1498.

[12] Zeng, H.C., Huang, S.H., Lai, S.H. (2008). Real-time video surveillance based on combining foreground extraction and human detection. Proc. 14th Int. Multimedia Modeling Conf., MMM 2008, Kyoto, Japan, 7079.

[13] Chen, Y.T., Chen, C.S. (2008). Fast human detection using a novel boosted cascading structure with meta stages. IEEE Trans. Image Process., 17(8), 1452-1464.

[14] Cheng, H.Y., Zeng, Y.J., Lee C.C., Hsu S.H. (2013). Segmentation of Pedestrians with Confidence Level Computation. Journal of Signal Processing Systems, 72(2), 87-97.

[15] Wang, X., Han, T. X., Yan, S. (2009). An HOG-LBP human detector with partial occlusion handling. Proc. IEEE Int. Conf. on Comput. Vision, ICCV 2009, Kyoto, 32-39.

[16] Geismann, P., Knoll, A. (2010). Speeding Up HOG and LBP Features for Pedestrian Detection by Multiresolution Techniques. Proc. 6th Int. Symposium Advances in Visual Computing, ISVC 2010, Las Vegas, NV, USA, 243-252.

[17] Zeng, C., Ma, H., Ming, A. (2010). Fast human detection using mi-sVM and a cascade of HOG-LBP features. Proc. 17th IEEE Int. Conf. on Image Processing (ICIP), 3845-3848.

[18] Crow, F. (1984). Summed-area tables for texture mapping. Proc. of SIGGRAPH, 18(3), $207-212$.

[19] Takagi, K., Tanaka, K., Izumi, S., Kawaguchi, H., Yoshimoto, M. (2014). A Real-time Scalable Object Detection System using Low-power HOG Accelerator VLSI. Journal of Signal Processing Systems.

[20] Jendernalik, W., Blakiewicz, G., Handkiewicz, A., Melosik, M. (2013). Analogue CMOS ASICs in image processing systems. Metrol. Meas. Syst., 20(4), 613-622.

[21] Everingham, M., Zisserman, A., Williams, C.K.I., Van Gool, L. (2006). The PASCAL Visual Object Classes Challenge 2006 (VOC 2006) Results. Technical Report, Univ. of Oxford.

[22] Chang, C.C., Lin, C.J. (2011). LIBSVM: a library for support vector machines. ACM Transactions on Intelligent Systems and Technology, 2(3) 27:1-27:27. 